\title{
Fluidic actuation for intra-operative in situ imaging
}

\author{
A. Devreker ${ }^{1}$, B. Rosa ${ }^{1}$, A. Desjardins ${ }^{2}$, E.J. Alles ${ }^{2}$, L.C. Garcia-Peraza ${ }^{2}$, E. Maneas ${ }^{2}$, \\ D. Stoyanov ${ }^{2}$, A.L. David ${ }^{3}$, T. Vercauteren ${ }^{2}$, J. Deprest ${ }^{4}$, S. Ourselin ${ }^{2}$, D. Reynaerts ${ }^{1}$, E. Vander Poorten ${ }^{1}$
}

\begin{abstract}
A novel fluidic actuation system has been developed for in situ imaging of anatomic tissues. The actuator consists of a micromachined superelastic tool guide driven by a pair of pneumatic artificial muscles. Two additional working channels allow easy interchange of instruments or sensing equipment. This paper describes the design and construction of the actuation system. Experimental results are also reported indicating a bending repeatability of 0.1 degrees and an operational bandwidth exceeding $8 \mathrm{~Hz}$. To show-case the performance of the device, the actuator was loaded with an all-optical ultrasound imaging probe. First scanned images of human placental tissue surface using an all-optical ultrasound probe are presented. While a model has been developed to estimate the probe position in space as function of the input pressure, in future work, this model will be complemented with additional sensor measurements of the bending probe taking into account the hysteretic behaviour of both muscles and nitinol structure.
\end{abstract}

\section{INTRODUCTION}

Minimal Invasive Surgery (MIS) offers faster patient recovery without affecting surgical outcome. While the demand to decrease the size and number of incisions in the patient grows, surgeons do not tolerate reduced functionality of their instruments. On the contrary, instruments should offer increased dexterity within the same or smaller package, allowing access to otherwise difficult to reach places deep in the body. These somewhat conflicting constraints fuel research into the design of novel surgical instrumentation. Many researchers investigate the offerings of less conventional actuators than e.g. cables, such as Shape-Memory Alloys (SMA) [1]-[4], magnetic steering [5], [6], concentric tube instruments [7], [8] or fluidic actuation [9]-[11].

It is the fluidic actuation technology which is explored within this paper. Pneumatic artificial muscles have been selected for several reasons. First, they offer a good force to volume ratio [12] and have low stiffness. This allows embedding these actuators into flexible structures resulting in active instruments with inherent compliance. This property is beneficial from a safety point of view when operating in complex and fragile environments. Through smart design this appealing property can be maintained even when expanding the number of DoFs (Degrees of Freedom) or the number of arms (e.g. in multi-arm instruments). Furthermore, pneumatic

\footnotetext{
1 KU Leuven, Department of Mechanical Engineering, 3001 Leuven, Belgium alain.devrekerekuleuven.be

${ }^{2}$ Department of Medical Physics \& Biomedical Engineering, University College London, United Kingdom

${ }^{3}$ UCL Institute for Women's Health, University College London, United Kingdom

${ }^{4}$ University Hospital Leuven, 3001 Leuven, Belgium
}

actuation can have similar bandwidth to hydraulic actuation and larger than SMA actuation, especially when miniaturized. This is important for example when operating in dynamic environments or when needing large speeds e.g. to efficiently scan large surfaces. Finally, these systems allow operation in large workspace. It is true that such muscles exhibit nonlinear hysteretic behaviour [13]-[15]. However, the pneumatic pressure lines powering the actuators do not affect the device's entire shape. This in contrast to tendon-driven actuators where friction between tendons and sheaths substantially depends on loading condition and actuator shape. Therefore, it should be possible to obtain good positioning precision and accuracy through appropriate modelling and closed-loop feedback with pneumatic actuation.

This paper presents a novel design of an actuator embedding pneumatic artificial muscles into a nitinol structure. The actuator design is presented in Section II. Section III presents the characterization of the actuator scanning abilities, and Section IV its modelling. Finally, Section V shows preliminary ex vivo validation experiments with human placenta, and Section VI concludes the paper and discusses further developments.

\section{DESIGN OF A NOVEL ACTUATOR FOR ENDOSCOPIC SCANNING}

This section presents the actuator design. At this point we opted for a modular system that allows studying the pneumatic performance actuation in detail through selecting and employing variety of sensors and exposing to different circumstances and loads, rather than optimizing for one particular application. The modularity allows us here to e.g. exchange instruments that are steered by the actuator. One application of special interest is for example the scanning of an imaging probe over a tissue surface. We are particularly interested in the following:

- the operating bandwidth. Bandwidth relates to scanning speed and acquisition time. Also, the interpretation of imaging simplifies if the effect of tissue motion during scans is reduced;

- the actuation repeatability. The higher the repeatability the better one can predict its behaviour. The images quality that are reconstructed using multiple subsequent measurements potentially increases as well;

- the miniaturization potential. This is crucial in order to maximally reduce invasiveness. Some operations such as fetal surgery require instrument diameters below 3 or 4 millimetres [16]; 
- the actuator model, in order to precisely predict the bending from the control input (i.e. the pressure, for a pneumatic actuator).
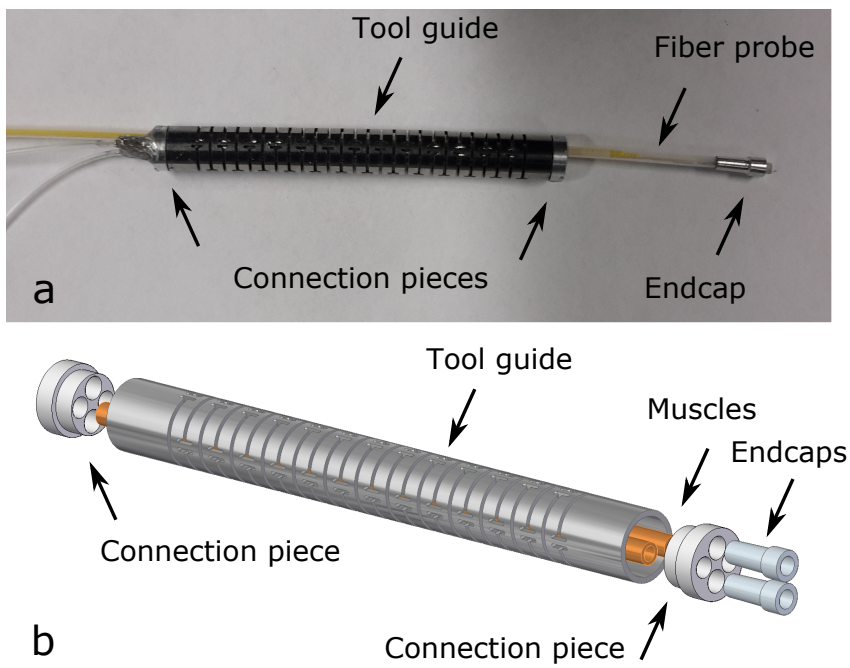

Fig. 1. Developed actuator made of a nitinol tool guide, 2 McKibben muscles and connections pieces: (a) actuator with probe inserted through it and fitted inside a conical endcap; (b) CAD drawing of the actuator.

\section{A. General layout of proposed actuator}

The proposed actuation system is depicted in Fig. 1. It consists of a nitinol tool guide that is bent by McKibben muscles. The tool guide has a structure similar to [17] and serves three purposes. First, it is machined in order to have a low bending stiffness and a high compression stiffness, making it suitable for a bending actuator. When pressurized, the McKibben muscles pull on the structure and cause it to bend. Second, the tool guide has a superelastic behaviour owing to its nitinol composition, it thus acts as return spring. When the muscles are not pressurized, the instrument will automatically come back to its neutral straight position. Finally, it also serves as an actuator outer sheath. Having this return spring externally also gives us maximal freedom to configure the internal part, which is convenient for building up a modular design.

The current design foresees four 'working channels', two of which are occupied by an antagonistic muscle pair, whereas the two others remain free. The total actuator length is $60 \mathrm{~mm}$ and its diameter is $7 \mathrm{~mm}$. This large diameter results from a modular design that was chosen here. Efforts to miniaturize the probe will be made in further study. Nevertheless, the fundamental behaviour of this actuation principle can be captured with current $7 \mathrm{~mm}$ design. Note that although the device is equipped with two antagonistic McKibben muscles, in this study, we only make use of a single muscle. The bending presented in the experiments will therefore be half the possible bending range of this actuator. This simplification is convenient as it allows us to ignore effects that arise when passing through the actuator 'neutral' (i.e. straight) pose. Additional modelling needed to capture this configuration is kept outside the scope of this paper.

\section{B. McKibben muscles}

Despite the non-linearities exhibited by pneumatic artificial muscles (PAMs), the latter are known and chosen for their good properties such as: high power/volume ratio, possible miniaturization, and inherent compliance. Different PAMs have been designed in the past [14], [18], [19]. In this study McKibben muscles were chosen for their fabrication simplicity and their smaller diameter when actuated, compared to other PAMs, e.g. pleated PAMs.

McKibben muscles are made of a balloon surrounded by a braided sleeve both attached to two sealing end connectors. When pressurizing the balloon, the surrounding sleeve constrains it and induces a muscle shortening. This contraction corresponds to the muscle pulling force. In-house made McKibben muscles are used here. The balloons are made from $1.96 \mathrm{~mm}$ outer diameter Silastic RX50 medical grade tubing (Dow Corning, USA). The surrounding sleeve consists of a metallic braided structure, with an outer diameter of $2.2 \mathrm{~mm}$ and a braid angle $\alpha$ of 29 degrees defined as the angle between braid fibres and the muscle longitudinal axis. The sealing is ensured by a two-component glue. The muscles are $52 \mathrm{~mm}$ long.

\section{Tool guide}

In order to transform the muscle contraction into bending motion, the muscles are integrated in an axially-stiff, bendable supporting structure, the so-called tool guide visible in Fig. 1. Superelastic nitinol was used to ensure low bending stiffness and elastic behaviour over large strains, similarly to [17]. Through wire-EDM (Electro Discharge Machining) a series of slots were made into a $56 \mathrm{~mm}$ long nitinol tube (outer, respectively inner, diameter of 7 and $6 \mathrm{~mm}$ ). The cutting pattern is designed so as to decrease the bending stiffness (in two directions) of the structure while minimally affecting compression stiffness. Figure 2(a) shows the dimensions of a single section/joint. Note that all joints remain connected, i.e. the tool guide remains a single piece. A mechanical stop is designed through FEM limiting the maximal stress upon each joint. These stops also limit the maximal bending angle per section (Fig. 2 (c)) to $\alpha \approx 7.2 \mathrm{deg}$. A total of 12 joints have been created leading to a total maximal bending angle of the tool guide of $\approx 84$ degrees in each direction.

\section{Connection pieces}

At both tool guide extremities, the actuator is enclosed by a pair of connecting pieces to which the muscles are clamped. The connection pieces have 4 holes to accommodate a pair of antagonistic McKibben muscles (2.2 mm diameter holes) and two remaining working channels of $2.3 \mathrm{~mm}$ allowing easy insertion of sensors and imaging devices. Special conical end-caps have been designed (Fig. 1) with varying inner diameters (1.7 to $1.9 \mathrm{~mm})$ for that purpose. The probes or sensors are inserted into the end-caps and can then easily be affixed or clicked into the distal tip by simply retracting the conical parts into the working channel (see Fig. 1). In this paper, an all-optical ultrasound probe is introduced in such a manner in one working channels (see Sec. V). This probe 


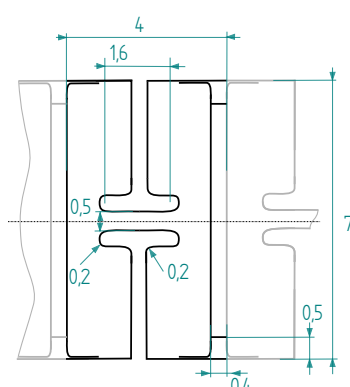

(a)

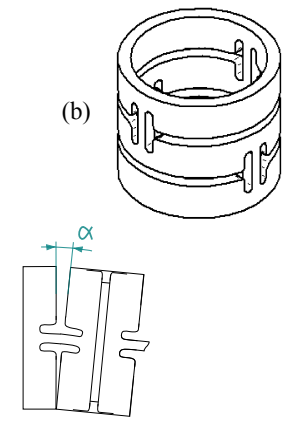

(c)
Fig. 2. (a) 2D CAD drawing of single joint of tool guide; (b) 3D representation; (c) maximal deformation $\alpha$ per joint. Units are in $\mathrm{mm}$.

could later, for example, be combined with a fibre bundle or a chip-on-tip camera, giving two imaging modalities within a single device. The camera and ultrasound images would then be co-localized at the end of the scanning device. This would simplify registering them to each other for advanced image treatment and overlay purposes.

\section{E. Pressure control}

The proposed actuation system is controlled by setting muscle internal pressure. A computer, running the realtime control framework OROCOS [20] is interfaced with an EtherCAT slave (National Instruments), which is equipped with an analog output module. The module voltage output drives a SMC ITV-0050 servovalve controlling the muscle pressure. Note that the servovalve uses an internal controller regulating the pressure with 0.01 bar accuracy. The valves output pressure was experimentally found to be stable in terms of amplitude up to $8 \mathrm{~Hz}$.

\section{EXPERIMENTAL CHARACTERIZATION OF THE ACTUATOR}

This section presents the basic performance of the proposed actuator. Experiments to derive the dynamic behaviour and the repeatability of the actuator have been conducted.

\section{A. Operational bandwidth}

In order to characterize the operational bandwidth of the actuating system, the actuator was tightened parallel to the imaging plane of a Prosilica AVT GC1290 camera. By limiting the size of the image field to $250 \times 200$ pixels, it was possible to increase the frame rate to $116 \mathrm{fps}$, thus making it possible to image the actuator even during fast motions. The camera was calibrated and the images were rectified to compensate for radial distortion. The actuator was then driven by a series of sine-wave pressure inputs with frequencies ranging from 0.1 to $8 \mathrm{~Hz}$. The amplitude of the pressure signals was set at 5 bars. Note that due to the limitations in switching speed of the available pressure valves, it was not possible to excite the device at frequencies exceeding $8 \mathrm{~Hz}$.

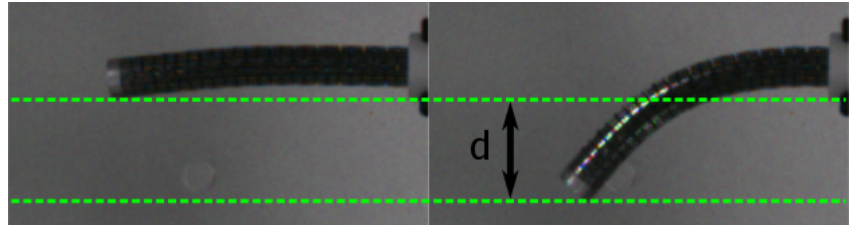

Fig. 3. Estimation of the amplitude $d$ spanned by the actuator at a given frequency. Left image is at neutral state, right at maximum bending, for a $0.1 \mathrm{~Hz}$ sine wave input. As it can be noticed on the left image, the actuator is pre-bent due to muscles imperfect alignment during assembly and internal muscle friction.

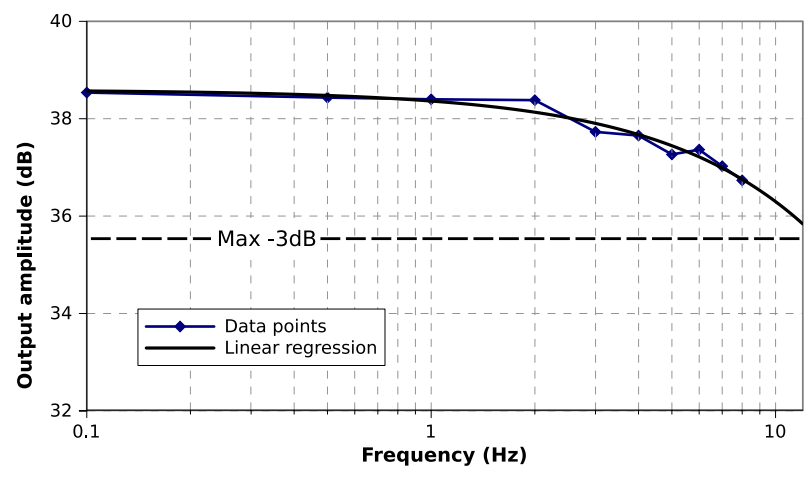

Fig. 4. Bode plot of the output amplitude in $\mathrm{dB}$

Figure 3 shows two snapshots at extreme positions during the excitation. First, the images corresponding to these extreme points were extracted from the image database. Then, the images were manually analysed and the distance $d$, as shown on Fig. 3, was extracted. Distance $d$ corresponds to the peak-to-peak amplitude at a given frequency. Recall that only a single muscle i.e. unidirectional motion is excited. This process was repeated over several excitation cycles to reduce the estimate inaccuracy that is introduced by manual measurement. Finally, the output amplitude in decibels is calculated. Fig 4 shows the bode plot of the measurements One can clearly see that the operational bandwidth is greater than $8 \mathrm{~Hz}$. The decrease in amplitude possibly originates in part from the limited valve performance, so further experiments need to be conducted with faster-switching valves to better characterize the bandwidth of the actuator itself. Nevertheless, a bandwidth $\geq 8 \mathrm{~Hz}$ is already very promising for applications where high-speed scanning is required with a minimally invasive instrument.

\section{B. Repeatability}

In order to estimate the repeatability two ElectroMagnetic (EM) sensors (Aurora, NDI Medical) were introduced in respectively the proximal and distal part of a working channel. The Aurora measurement system measures at 40 $\mathrm{Hz}$ the absolute pose (position and orientation) of the EMsensors with respect to the location of the electromagnetic field generator. Care was paid to minimize the EM field distortion by removing metallic objects from the measurement volume. Note that the metallic parts present in the actuator itself, aluminium and nitinol in this case, are paramagnetic 
materials. These materials have an extremely low magnetic permeability. Their effect on the measurement precision and accuracy of the NDI Aurora system has previously been found to be negligible [21].

With the aim of this measurement being the actuation repeatability estimation, the precision of the Aurora measurement system is of greater concern than its accuracy, especially since we aim at comparing relative position and orientation measurements in a small volume. Nafis et al. reported in [21] a static precision of $0.03 \mathrm{~mm}$ for the Aurora $6 \mathrm{DoF}$ tracking system, increasing to $0.26 \mathrm{~mm}$ for a probe moving at $50 \mathrm{~mm} / \mathrm{s}$. However as in these experiments, we impose a $0.1 \mathrm{~Hz}$ sine wave to the actuator, its tip moves at a speed of approximatively $4 \mathrm{~mm} / \mathrm{s}$, so it is safe to assume accuracies close to the static case.

In order to measure the actuator bending, the EM probe was first inserted in the proximal side of the working channel. Its unit vector $\mathbf{v}_{p}$ pointing outwards the actuator base position has been measured. Then, the probe has been attached to the distal tip using the conical end-caps previously described. The bending angle $\theta$ was then estimated as the angle between the proximal tip vector $\mathbf{v}_{t}$ and $\mathbf{v}_{p}: \theta=\mathbf{v}_{t}^{T} \mathbf{v}_{p}-\theta_{0}$, where $\theta_{0}$ is a constant offset angle at rest position $(P=0$ bar $)$ accounting for initial misalignment of proximal and distal EM sensor.

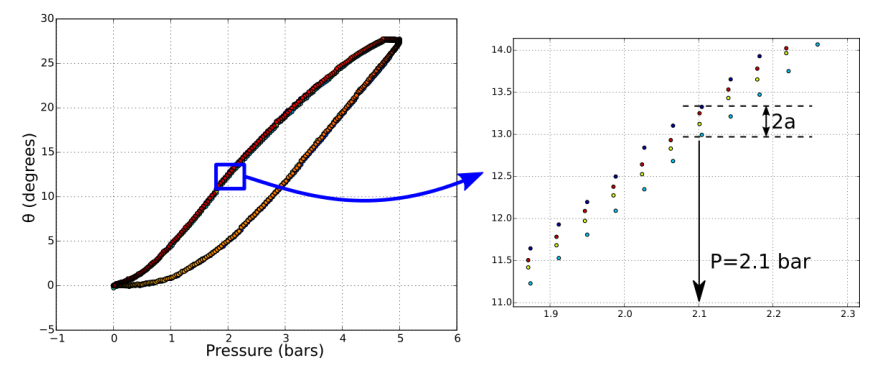

Fig. 5. Pressure vs. angle graph as measured by the aurora. The right part zooms in on an example section of this graph. It shows the variation $a$ estimated over four cycles. Here a section with pressure of 2.1 bar, in the upper part of the hysteresis cycle is selected, but other sections show similar behaviour.

A $0.1 \mathrm{~Hz}$ pressure sine wave, ranging from 0 to 5 bars, was applied as input. For each cycle, the bending angle $\theta$ was estimated. The repeatability is then defined as the amplitude $a$ of the variation of $\theta$ between different cycles for the same pressure input (see Fig. 5). The angle output repeatability was computed for four cycles and was found to be on average 0.1 degrees, with a standard deviation of 0.05 degrees. This makes an average repeatability of $0.17 \mathrm{~mm}$ for tangential displacement of the actuator tip (standard deviation $0.10 \mathrm{~mm}$ ). Though the actuator exhibits a clear hysteretic behaviour, as can be seen on Fig. 5, this result shows that a specific pressure input will lead to a very repeatable movement, irrespective of the shape of the hysteresis curve.

\section{Modelling}

This section establishes the control that is used in order to precisely control the tip movement. It is at this stage an open loop control based on a actuator model.

\section{A. Modelling of the actuator}

In order to establish an actuator model, one has to link the controller output, i.e. the pressure, to the actuator displacement or bending. This is done by combining a model of the actuator's structure using common models used for continuum robots with a model of the McKibben muscles themselves.

The muscle model considered in this study is the model established by Gaylord [22]:

$$
F_{G}=\frac{P}{4 \pi N^{2}}\left(3 L^{2}-B^{2}\right)
$$

Where $F_{G}$ is the McKibben muscle output force depending on the pressure $P$, the number of turns $N$ of a braid fibre around the muscle, the length $B$ of such a fiber, and the muscle length $L . P$ is the input, $N$ and $B$ are constants, and $L$ varies with force and pressure. In order to link it to the pressure, one can consider the actuator structure including the tool guide as a simple beam of bending modulus $E I$ and bending radius $\rho$. The Euler-Bernoulli beam theory says that, for small deflections, the bending moment $M_{E B}$ is then:

$$
M_{E B}=\frac{-E I}{\rho}
$$

The bending moment and the moment exerted by the muscle cancel each out leading to an equilibrium state which, for the muscle force $F_{G}$ acting at a lever distance $d$ from the center, can be expressed as $M_{E B}+F_{G} d=0$. By inserting (1) into this equation, one can then solve for $L$ :

$L=\frac{2 \pi N^{2}}{3 P}\left(-\frac{E I}{d^{2} L_{0}}+\sqrt{\left(\frac{E I}{d^{2} L_{0}}\right)^{2}+\frac{3 P}{N^{2} \pi}\left(\frac{P B^{2}}{4 N^{2} \pi}+\frac{E I}{d^{2}}\right)}\right)$

This equation can then be used for computing the bending angle $\theta$ by noticing that if constant curvature is assumed, $\theta=\frac{L_{0}-L}{d}$.

\section{B. Model corrections}

McKibben muscles are known for their nonlinear behaviour, which is confirmed by first experimental results presented in Fig. 5. In order to model this nonlinear behaviour and negative effects not considered in the Gaylord force expression, three parameters were added to the model.

$P_{d b}$ is a parameter representing a pressure deadband. When the muscle is not pressurized, the balloon diameter is smaller than the braid inner diameter requiring a certain amount of pressure before the balloon touches the braid and the muscle starts contracting. $L_{t i p}$, defined as the length of the non-active muscle ends, compensates for the muscle conical tip effect [15]. The theoretical muscle length is thus to be reduced by $2 L_{t i p}$.

Finally, friction between the balloon and the sheath, as well as between the sheath and the tool guide reduce the bending range. We chose here to model it as a static friction of value $F_{c}$. At every pressure input sign change, the movement stops because of friction. When the model 
output force $F$ (see eq. 1) overcomes the threshold $F_{c}$, the actuator starts moving again. Note that at the start, the static friction must also be overcome.

\section{Parameters identification}

Numerous model parameters are known by design, such as the muscle length at rest $L_{0}=52 \mathrm{~mm}$, the braid angle $\alpha=29$ degrees, and the lever $d=1.6 \mathrm{~mm}$. Knowing $\alpha$, the number of turns $N$ and the fibre length $B$ are computed from an helix equation, using as diameter the braid diameter $2.1 \mathrm{~mm}$. $L_{t i p}$ has been estimated at $2 \mathrm{~mm}$ based on actuated muscle tip shape measurements.

$P_{d b}$ was estimated as the pressure at which the actuator starts moving, estimated at 0.35 bar. $F_{c}$ was estimated at a value of $1.23 \mathrm{~N}$ so that the width of the exhibited hysteresis was similar to the one experimentally measured (see Fig. 5).

Finally, the bending stiffness $E I$ has been experimentally measured by deflecting the structure and simultaneously measuring the applied force and resulting deflection with a force and laser distance sensor respectively. A motor was used to deflect the structure by steps of $0.1 \mathrm{~mm}$. In such a way an average bending stiffness of $E I=0.6010^{-3} \mathrm{Nm}^{2}$ was estimated.

\section{Experimental validation}

Fig. 6 presents the model output bending angle $\theta_{\text {mod }}$, compared to the angle $\theta_{\text {exp }}$ measured with the Aurora system. The error $e=\theta_{\text {exp }}-\theta_{\text {mod }}$ is presented on the right. The modelling error $e$ is on average 0.48 degrees, with a standard deviation of 1.6 degrees. Considering the simplicity of the model used in this study, this is a promising result. Further studies will look in greater detail at more complex non-linear modelling techniques in order to more accurately predict the actuator bending.
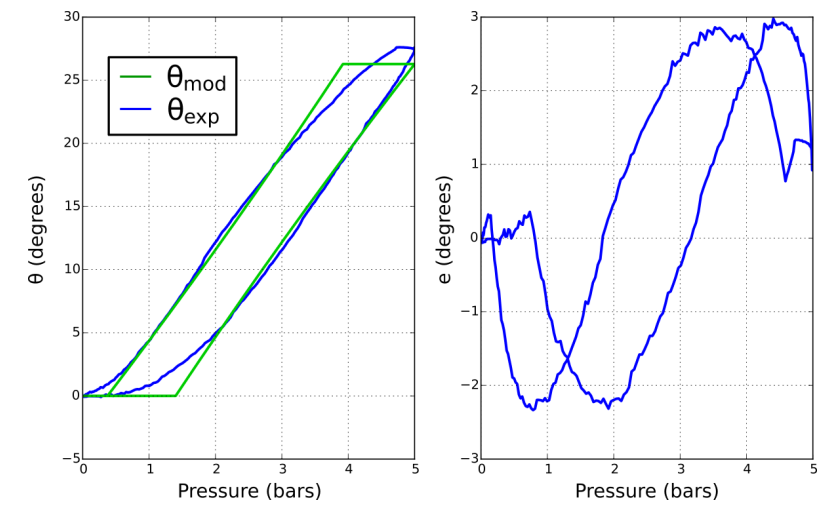

Fig. 6. Comparison between model and experimental measures. Left: bending angle vs. pressure. Right: evolution of $e$.

\section{E. Design of future actuators}

We have previously shown that the output angle predicted by the model is close to the measured one (see Fig. 6). As mentioned in the introduction, the aim of this paper is to propose a modular design that can be used for testing and evaluation. More compact actuators, dedicated to particular applications, will be made in the future. An already interesting use of the proposed model is to employ it for designing such actuators. By varying the different model parameters, one can for example estimate the maximum bending angle for particular parameters or instruments inside the working channels.

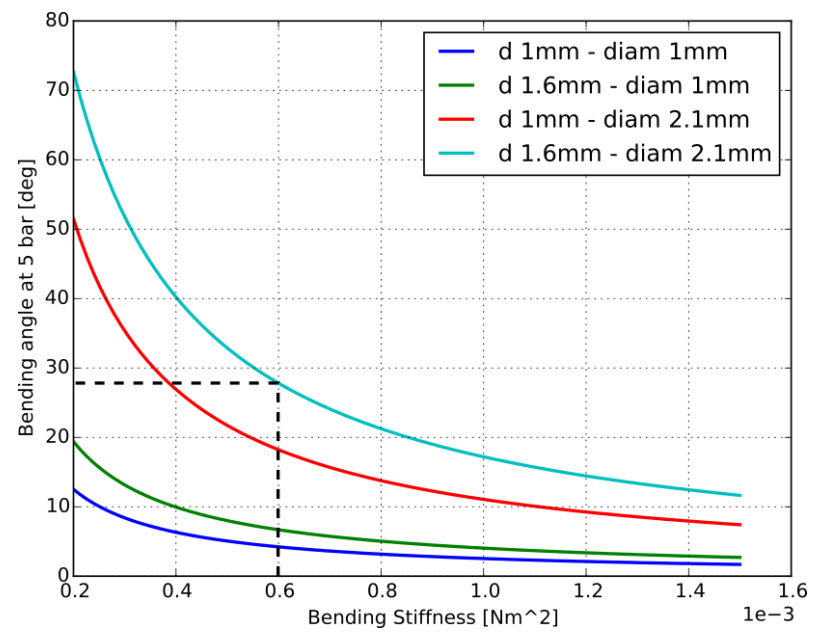

Fig. 7. Maximum bending angle at 5 bar depending of the actuator stiffness for different model parameters. The dashed line represents the current actuator.

Fig. 7 shows the maximum bending angle at 5 bars in function of the actuator composite stiffness for different values of lever $d$ and McKibben muscle diameter. Given certain desired actuator outer diameters and working channel, one can then estimate the required nitinol tool guide stiffness for a desired output angle. This results can then be used to choose an appropriate nitinol tube thickness as well as a wire-EDM cutting pattern (see Sec. II-C).

\section{Ex vivo ALL-OPTICAL ULTRASOUND IMAGING OF HUMAN PLACENTA}

This section describes the use of the novel actuator to image a human placenta through optical ultrasound. Optical ultrasound, in which ultrasound is generated and received with light, is a promising modality for minimally invasive surgery. With this modality, ultrasound is generated using the photoacoustic effect, with modulated excitation light provided to an optically absorbing coating. Ultrasound that reflects from tissue structures can be received optically using a Fabry-Pérot etalon for pulse-echo sensing. In this study, both the optically absorbing coating and the Fabry-Pérot etalon were positioned at the distal ends of optical fibres.

The optical ultrasound probe was created with the ultrasound generating and receiving fibres; an additional fibre with a bare end delivered modulated light ahead of the probe so that ultrasound could be generated within tissue for photoacoustic sensing, and to provide a guide beam of light to tissue. Given its flexibility and small dimensions that are compatible with the developed actuator (outer diameter: $1.9 \mathrm{~mm}$ ), this probe is particularly well suited for imaging in confined spaces deep in the body, in close proximity 
to anatomical structures. With two mechanisms generating ultrasound, this probe provides contrast for tissue morphology with pulse-echo ultrasound sensing, and contrast for blood vessels with photoacoustic sensing. Each mechanism generates a series of A-lines that are concatenated in realtime to form an M-mode image as the McKibben actuator is moving.

A promising application for this sensing technology is fetal surgery, and especially the Twin-to-Twin Transfusion Syndrome, in which anastomoses at the placenta level between monochorionic twins have to be photocoagulated under fetoscopic visualization by the use of a laser [16]. Anastomoses between subsurfacic blood vessels are hard to identify in endoscopic images and are a possible source of intervention failure [23]. Therefore, a depth-resolved imaging modality such as ultrasound imaging would be highly beneficial for the surgery outcome. However, ultrasound images acquired from the outside of the body are not sufficiently precise to identify subsurfacic structures at the placenta level. In the following we present some results of ex vivo scans carried out with the optical ultrasound probe and the actuator.

\section{A. Imaging of single wire phantom}

A simple phantom was first imaged in order to test the system performance. The phantom consisted of the stylet of a 20 gauge needle (diameter $0.6 \mathrm{~mm}$ ) placed into a bath of water for reflecting ultrasound waves. The imaging probe was placed into one of the actuator working channels. The actuator was then positioned over the phantom so that the scanning direction was perpendicular to the stylet.

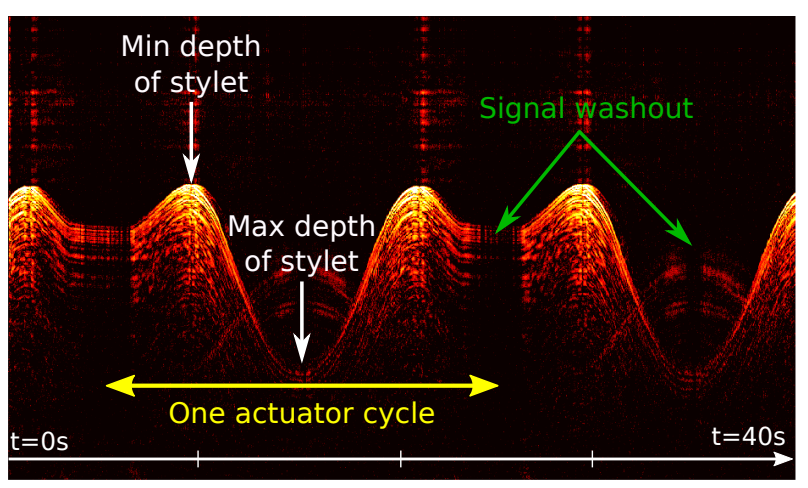

Fig. 8. M-mode image acquired with an all-optical ultrasound probe and the moving actuator over the stylet of a 20 gauge needle.

Fig. 8 shows the result of a 40 seconds scan. The actuator input pressure was a sine wave signal of $0.05 \mathrm{~Hz}$, with a maximum pressure of 4 bars. The M-mode image is the concatenation over time of A-line scans acquired by the sensing probe $(50 \mathrm{~Hz}$ A-line acquisition rate for a total of 2000 A-lines). The imaging depth is $30 \mathrm{~mm}$. To suppress cross-talk between transmit and receive fibres, a dynamic background substraction algorithm is applied to the signal. This results in signal washout at the end of the actuator stroke when it slows down (the input is a sine wave). In the future, the actuator trajectory knowledge will be integrated in the background suppression algorithm in order to avoid this effect.

\section{B. Imaging of human placenta}

In order to demonstrate the applicability for the targeted clinical use, a term human placenta collected at caesarean section birth was imaged (ethical approval UCL 08/H0714/87). Following the same protocol as for the phantom scan, the fibered imaging probe was introduced into the actuator and placed in front of the fetal placental surface. The placenta was placed in a water tank with maternal side down and the fluid covering the fetal placental surface (see Fig. 9). This is similar to the real clinical situation in which the probe inside the actuator would be inserted via a trocar into the amniotic sac, which is filled with amniotic fluid.

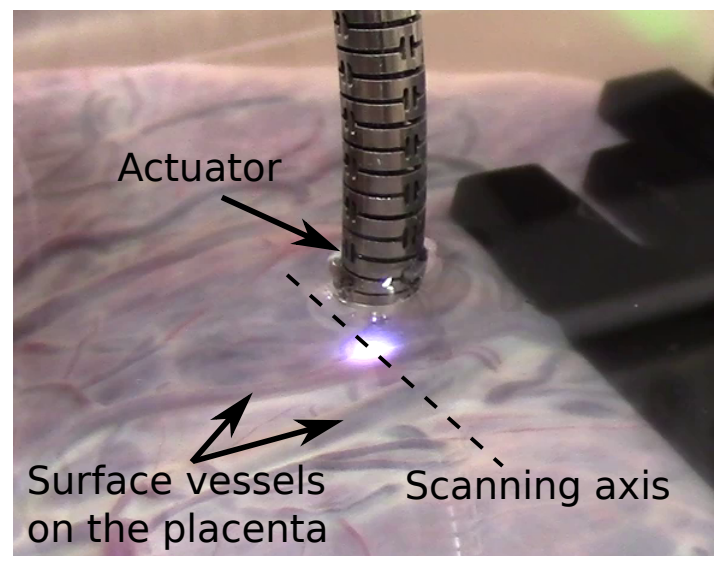

Fig. 9. Picture of the experimental setup with ex vivo experiments, showing surface vessels of fetal side of the placenta, and the actuator. The imaging probe is fixed inside one of the actuator working channels. The white-light spot on the placenta is a projected guide beam for illustration only.

Fig. 10 shows the M-mode image resulting from a $20 \mathrm{sec}-$ onds scan. One can clearly see that both surfacic and subsurfacic features of tissue are identifiable on the image. This result shows the potential of a combination of the pneumatic actuation technology, together with all-optical ultrasound imaging, for intra-uterine in situ imaging of the placenta.

\section{CONCLUSION}

This paper presents an actuator for in situ imaging scanning. McKibben muscles are embedded into a superelastic Nitinol tool guide. The actuator is driven by pressure and exhibits an operational bandwidth larger than $8 \mathrm{~Hz}$. The average repeatability for a tangential displacement of the tip is very good, with an average of $0.17 \mathrm{~mm}$ and a standard deviation of $0.10 \mathrm{~mm}$.

A model is proposed to be able to predict the bending in function of a given input pressure. This model makes use of an Euler-Bernoulli beam equation composed together with the Gaylord expression of the force delivered by a McKibben muscle. Correction factors for pressure deadband and hysteresis due to friction inside the actuator are added, resulting in a low prediction error (standard deviation 1.6 degrees over a full 0-5-0 bars cycle). Furthermore, it is shown that this 


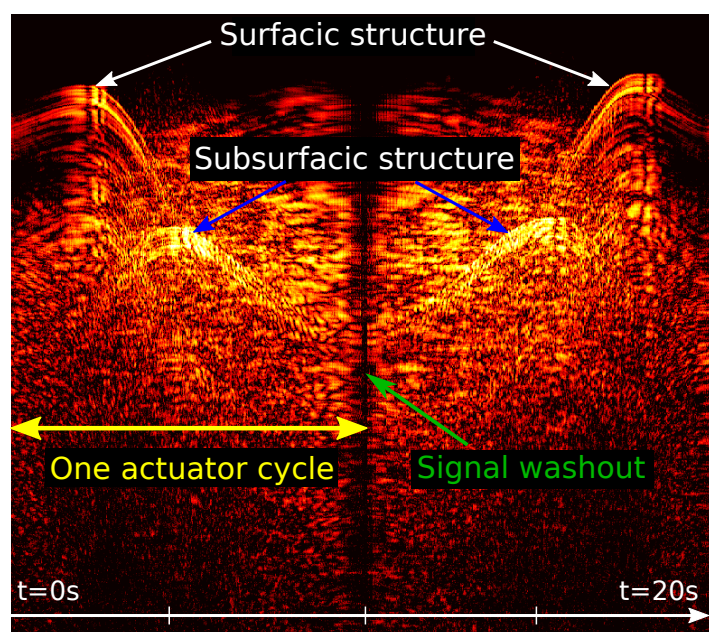

Fig. 10. M-mode image acquired with an all-optical ultrasound probe and the moving actuator over the fetal surface of a human placenta.

model can be used for design parameters optimization of future actuators, and particularly for the design of the nitinol tool guide and its desired stiffness.

Finally, as first application, the actuator is used for scanning with an all-optical ultrasound probe, an imaging modality that is particularly promising for use in intrauterine fetal surgery. A fibre probe is inserted into the actuator and scans are made, first on a phantom then on a human placenta. M-mode images acquired show the proof of concept. Since M-mode images may be difficult to interpret for clinicians in some contexts, reconstruction of 2D images is however needed. This is a challenging problem as the actuator movement with respect to the tissue is unknown.

Future research will look in three directions. First, new versions of the actuator with updated design parameters for specific applications will be made. Hydraulic actuation will be further investigated. Second, the actuator modelling will be refined in order to take into account higher order behaviour induced by friction and nonlinear effects. Finally, actuator tracking and fusion with the actuator model will be investigated in order to reconstruct $2 \mathrm{D}$ and 3D PA/US images in real time.

\section{ACKNOWLEDGEMENT}

This work was supported through an Innovative Engineering for Health award by the Wellcome Trust [WT101957]; Engineering and Physical Sciences Research Council (EPSRC) [NS/A000027/1]. ALD is supported at UCLH/UCL by funding from the Department of Health NIHR Biomedical Research Centres funding scheme. AD, EVP and BR partially funded by the European Commission FP7-ICT Programme, CASCADE project under grant agreement No. 601021.

\section{REFERENCES}

[1] K. Ikuta, M. Tsukamoto, and S. Hirose, "Shape memory alloy servo actuator system with electric resistance feedback and application for active endoscope," in IEEE Int. Conf. on Robotics and Automation, 1988, pp. 427-430.
[2] T. Fukuda, S. G., K. Kosuge, F. Arai, M. Negoro, and K. Nakabayashi, "Micro active catheter system with multi degrees of freedom," in IEEE Int. Conf. on Robotics and Automation, may 1994, pp. 2290 -2295.

[3] D. Reynaerts, J. Peirs, and H. Van Brussel, "Shape memory microactuation for a gastro-intestinal intervention system," Sensors and Actuators, vol. 77, pp. 157-166, 1999.

[4] Y. Haga, T. Mineta, W. Makishi, T. Matsunaga, and M. Esashi, "Active bending catheter and electric endoscope using shape memory alloy," Shape Memory Alloys, Corneliu Cismasiu (Ed.),, pp. 1-21, 2010.

[5] Y. Fu, H. Liu, W. Huang, S. Wang, and Z. Liang, "Steerable catheters in minimally invasive vascular surgery," Int. J. Med. Robotics and Comput. Assist. Surg., vol. 5, no. 4, pp. 381-391, 2009.

[6] A. Menciassi, P. Valdastri, C. Quaglia, E. Buselli, and P. Dario, "Wireless steering mechanism with magnetic actuation for an endoscopic capsule," in IEEE/EMBS Conf. on Engineering in Medicine and Biology (EMBC), 2009, pp. 1204-1207.

[7] R. Webster, A. Okamura, and N. Cowan, "Toward active cannulas: Miniature snake-like surgical robots," in IEEE/RSJ Int. Conf. on Intelligent Robots and Systems, 2006, pp. 2857-2863.

[8] P. Sears and P. Dupont, "A steerable needle technology using curved concentric tubes," in IEEE/RSJ Int. Conf. on Intelligent Robots and Systems, 2006, pp. 2850-2856.

[9] K. Ikuta, H. Ichikawa, and K. Suzuki, "Safety-active catheter with multiple-segments driven by micro hydraulic actuators," in Proc. Medical Image Computing and Computer-Assisted Intervention, 2002, pp. 182-191.

[10] B. Gorissen, M. De Volder, A. De Greef, and D. Reynaerts, "Theoretical and experimental analysis of pneumatic balloon microactuators," Sensors and Actuators A, Physical, vol. 168, no. 1, pp. 58-65, 2011.

[11] A. Moers, M. De Volder, and D. Reynaerts, "Integrated high pressure microhydraulic actuation and control for surgical instruments," Biomedical Microdevices, pp. 1-10.

[12] A. Devreker, E. Vander Poorten, P. Tran, H. De Praetere, P. Herijgers, J. Vander Sloten, and D. Reynaerts, "Towards fluidic actuation for catheter-based interventions," in Proceedings Actuator 2014, 2014, pp. $173-176$.

[13] B. Tondu and P. Lopez, "Modeling and control of mckibben artificial muscle robot actuators," Control Systems, IEEE, vol. 20, no. 2, pp. 15-38, 2000.

[14] E. Hocking and N. Wereley, "Analysis of nonlinear elastic behavior in miniature pneumatic artificial muscles," Smart Materials and Structures, vol. 22, no. 1, p. 014016, 2013.

[15] C. Kothera, M. Jangid, J. Sirohi, and N. Wereley, "Experimental characterization and static modeling of mckibben actuators," Journal of Mechanical Design, vol. 131, no. 9, p. 091010, 2009.

[16] M.-V. Senat, J. Deprest, M. Boulvain, A. Paupe, N. Winer, and Y. Ville, "Endoscopic laser surgery versus serial amnioreduction for severe twin-to-twin transfusion syndrome," New England Journal of Medicine, vol. 351, no. 2, pp. 136-144, 2004.

[17] J. Peirs, D. Reynaerts, H. Van Brussel, G. De Gersem, and H.-W. Tang, "Design of an advanced tool guiding system for robotic surgery," in IEEE Int. Conf. on Robotics and Automation, 2003, pp. 2651-2656.

[18] D. Villegas, M. Van Damme, B. Vanderborght, P. Beyl, and D. Lefeber, "Third-generation pleated pneumatic artificial muscles for robotic applications: Development and comparison with mckibben muscle,' Advanced Robotics, vol. 26, no. 11-12, pp. 1205-1227, 2012.

[19] R. Niiyama and Y. Kuniyoshi, "Enhanced design of a pneumatic artificial muscle for musculoskeletal robot: Super long stroke pneumatic muscle for articular mechanisms," in Proc. ICRA 2011 Workshop on Biologically-Inspired Actuation, 2011, pp. 13-14.

[20] H. Bruyninckx, P. Soetens, and B. Koninckx, "The real-time motion control core of the Orocos project," in IEEE Int. Conf. on Robotics and Automation, 2003, pp. 2766-2771.

[21] C. Nafis, V. Jensen, L. Beauregard, and P. Anderson, "Method for estimating dynamic em tracking accuracy of surgical navigation tools," in Medical Imaging, 2006, pp. $61410 \mathrm{~K}-61410 \mathrm{~K}$.

[22] R. H. Gaylord, "Fluid actuated motor system and stroking device," Jul. 22 1958, uS Patent 2,844,126.

[23] L. Lewi, J. Jani, M. Cannie, Y. Robyr, R.and Ville, K. Hecher, E. Gratacos, H. Vandecruys, V. Vandecaveye, S. Dymarkowski et al., "Intertwin anastomoses in monochorionic placentas after fetoscopic laser coagulation for twin-to-twin transfusion syndrome: is there more than meets the eye?" Am. J. of obstetrics and gynecology, vol. 194, no. 3, pp. 790-795, 2006. 